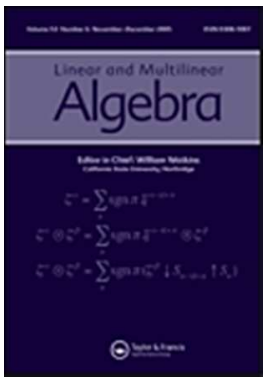

\title{
Evolution coalgebras on chicken populations
}

\begin{tabular}{|r|l|}
\hline Journal: & Linear and Multilinear Algebra \\
\hline Manuscript ID & 2018-0205.R1 \\
\hline Manuscript Type: & Original Article \\
\hline Date Submitted by the Author: & n/a \\
\hline Complete List of Authors: & $\begin{array}{l}\text { Paniello, Irene; Public University of Navarre, } \\
\text { Statistics and Operative Research }\end{array}$ \\
\hline $\begin{array}{r}\text { Keywords: } \\
\text { href="http://www.ams.org/mathscinet/msc/msc2010.html" } \\
\text { target="_blank" }>2010 \text { Mathematics Subject } \\
\text { Classification }</ a>:\end{array}$ & $\begin{array}{l}\text { Evolution coalgebra, Chicken population, } \\
\text { Digraph }\end{array}$ \\
\hline $\begin{array}{r}\text { 17D92, 16T99, 16W99 } \\
\hline\end{array}$ & \\
\hline
\end{tabular}

SCHOLARONE ${ }^{\mathrm{m}}$

Manuscripts 
This is an accepted manuscript of an article published by Taylor \& Francis in Linear and Multilinear Algebra on 20 Aug 2018, available online: https://doi.org/10.1080/03081087.2018.1508408

\title{
Evolution coalgebras on chicken populations
}

\section{Paniello}

Department of Statistics and Operative Research, Public University of Navarre, Pamplona, Spain

\author{
ARTICLE HISTORY \\ Compiled July 20, 2018
}

\begin{abstract}
We introduce a coalgebra structure for chicken populations, the evolution coalgebra of a chicken population, and review its main properties. A notion of simplicity is also considered together to a characterization of simple coalgebras of chicken populations given in terms of the strongly connectedness of their attached digraphs, a notion also introduced here.
\end{abstract}

\section{KEYWORDS}

Evolution coalgebra; Chicken population; Digraph

\section{AMS CLASSIFICATION \\ 17D92; 16T99; 16W99}

\section{Introduction}

The coalgebraic approach to genetic populations was firstly considered by Tian and Li [17], for populations ruled by Mendel's laws, aimed to provide an algebraic model to describe the backwards inheritance of the genetic information between generations. The cubic matrix representation of genetic coalgebras, later considered in [8], contributed to solve some of the question that remained open in [17] setting also a connection between genetic coalgebras and Markov processes [9]. The structure of genetic coalgebras has been recently studied in terms of the strongly connectedness of their attached oriented trigraphs [11].

On the other hand, although genetic populations not obeying Mendel's laws, but based on the self-replication of their individuals, have been till now mainly described in terms of evolution algebras (we refer the reader to Tian's foundation of evolution algebras given in [16]), it has been recently shown that considering such populations from the coalgebraic viewpoint is also possible [13].

Here we attempt to extend that point of view considered in [13] to chicken populations. Chicken populations were described by Labra, Ladra and Rozikov in [4] (see also [6]) as a simplified example of bisexual populations [5] with only one existing male type. Such a reduction, from bisexual to chicken populations, is mainly motivated by the complexity of the cubic structure matrices that appear attached to bisexual populations. These structure matrices become much simpler square matrices under the 


\section{Chicken populations.}

Evolution algebras of chicken populations were introduced in [4] (see also [6]) as a particular case of evolution algebras for bisexual populations [5] where only one male type (called roaster) is allowed. In what follows, unless otherwise stated, we will consider $K$ to be a field of characteristic not 2 .

Definition 2.1. [4, Definition 2.1] An evolution algebra of a chicken population (EACP for short) is a $(n+1)$-dimensional $K$-algebra $\mathcal{C}$ with (natural) basis $\mathcal{B}=$ $\left\{h_{1}, \ldots, h_{n}, r\right\}$ and multiplication given by $h_{i} r=r h_{i}=\frac{1}{2}\left(\sum_{j=1}^{n} a_{i j} h_{j}+b_{i} r\right), i=$ $1, \ldots, n$, and zero otherwise (i.e. $h_{i} h_{j}=h_{j} h_{i}=0$ for all $i, j=1, \ldots, n$ and $r^{2}=0$ ).

Reduction from bisexual to chicken populations is aimed to obtain a more easy to handle matrix representation of the structure constants attached to the algebra multiplication. Indeed the structure constants of any $(n+1)$-dimensional EACP can be gathered into a $n \times(n+1)$ matrix

$$
\left(\begin{array}{ccc|c}
a_{11} & \cdots & a_{1 n} & b_{1} \\
\vdots & \ddots & \vdots & \vdots \\
a_{n 1} & \cdots & a_{n n} & b_{n}
\end{array}\right)
$$

EACPs of arbitrary dimension were also considered in [4, Definition 2.1].

As noted in [4], it follows from [5, Theorem 4.1] that, EACPs are commutative, but not associative or even power-associative in general. Moreover EACPs are not unital [4, 
Proposition 2.14]. Subalgebras of EACPs were also introduced in [4], following Tian's notion of evolution subalgebras [16], and later studied in [7]. EACPs of dimension 2 and also those 3-dimensional EACPs with $\operatorname{dim}_{K}\left(\mathcal{C}^{2}\right) \neq 1$ were classified in [4, Proposition 6.1, Theorem 6.2]. A complete classification of 3-dimensional complex EACPs was later given in [2].

\section{Coalgebras.}

We refer the reader to [15] for basic results on coalgebras. See also [13] for those notions more closely related to coalgebras appearing in genetics.

\subsection{Coalgebras.}

A coalgebra $C$ is a $K$-vector space with a linear map $\Delta: C \rightarrow C \otimes C$ called comultiplication. A coalgebra $(C, \Delta)$ is coassociative if $(\Delta \otimes i d) \Delta=(i d \otimes \Delta) \Delta$ and cocommutative if $\tau \Delta=\Delta$, where $\tau: C \otimes C \rightarrow C \otimes C$ denotes the twist map $\tau(a \otimes b)=b \otimes a$ for all $a, b \in C$. A coalgebra $(C, \Delta)$ is counital if it is endowed with a linear map (counit) $\varepsilon: C \rightarrow K$ such that $(i d \otimes \varepsilon) \Delta=(\varepsilon \otimes i d) \Delta=i d$.

\subsection{Subcoalgebras and coideals.}

Let $(C, \Delta)$ be a coalgebra. A subspace $D$ of $C$ is a subcoalgebra if $\Delta(D) \subseteq D \otimes D$. A subspace $I$ of $C$ is a left coideal (resp. a right coideal) if $\Delta(I) \subseteq C \otimes I$ (resp. if $\Delta(I) \subseteq I \otimes C$ ) and a coideal if $\Delta(I) \subseteq I \otimes C+C \otimes I$ (and $\varepsilon(I)=0$ if $C$ is counital). Given a subset $S$ of a coalgebra $C$ we denote by $\operatorname{subcoalg}(S)$ the subcoalgebra of $C$ generated by $S$, that is, the intersection of all coalgebras of $C$ containing $S$.

Example 3.1. The dual vector space $A^{*}=\operatorname{Hom}_{K}(A, K)$ of any finite-dimensional associative $K$-algebra $A$ with multiplication $m: A \otimes A \rightarrow A$, has a coalgebra structure $\left(A^{*}, \Delta\right)$ given by $\Delta=\rho^{-1} m^{*}$, where $\rho$ denotes the isomorphism $A^{*} \otimes A^{*} \cong(A \otimes A)^{*}$. Here $\rho$ is an isomorphism by the finite-dimensionality of $A$ [15]. We remark here that the coalgebra structure on $A^{*}$ needs of the finite-dimensionality of $A$. Otherwise the finite dual $A^{\circ}$ should be considered. However the dual vector space $C^{*}$ of any coassociative coalgebra $C$ can always be endowed with an associative algebra structure [15, Proposition 1.1.2].

\subsection{Baric coalgebras.}

A baric coalgebra $(C, \Delta, \phi)$ is a (non-necessarily counital) coalgebra $(C, \Delta)$ with a character $\phi$, that is, a nonzero linear map $\phi: C \rightarrow K$ such that $(\phi \otimes \phi) \Delta=\phi$. Characters of the coalgebra $C$ are the idempotents of its dual algebra $C^{*}[10$, Proposition $1]$.

\subsection{Coalgebras with genetic realization.}

The notion of coalgebra with genetic realization was considered in [17] for Mendelian populations. A (finite-dimensional) real coalgebra $C$ has genetic realization if it has a (natural) basis $\mathcal{B}=\left\{e_{1}, \ldots, e_{n}\right\}$ such that the comultiplication constants of the 
basis elements are nonnegative and satisfy $\sum_{i, j=1}^{n} \beta_{i j}^{k}=1$ for all $k=1,2, \ldots, n$, where $\Delta\left(e_{k}\right)=\sum_{i, j=1}^{n} \beta_{i j}^{k} e_{i} \otimes e_{j}$ for all $k=1,2, \ldots, n$. Examples of coalgebras with genetic realization can be found in [10].

Coalgebras with genetic realization are not necessarily coassociative or cocommutative, but can admit characters. Their characters were described in [10]. The study of coalgebras with genetic realization has been based on their connection to cubic matrices with stochastic properties [8].

\subsection{Evolution coalgebras.}

A new model of coalgebra motivated by self-reproduction processes occurring in nonmendelian populations has been recently introduced in [13]. An evolution coalgebra is a $K$-coalgebra (here $K$ denotes any field) having a (natural) basis $\mathcal{B}=\left\{e_{1}, \ldots, e_{n}\right\}$ with comultiplication $\Delta\left(e_{k}\right)=\sum_{i=1}^{n} \beta_{i i}^{k} e_{i} \otimes e_{i}$ for all $k=1,2, \ldots, n$. To endow any (real) evolution coalgebra with genetic realization it then suffices to require the nonnegativity of the $\beta_{i i}^{k}$ 's and also $\sum_{i=1}^{n} \beta_{i i}^{k}=1$ for all $i, k=1,2, \ldots, n$.

\section{Coalgebras on chicken populations.}

In this section, following [13], we introduce a coalgebraic framework for chicken populations. All coalgebras considered here are defined over a field $K$ of characteristic not 2 .

Definition 4.1. An evolution coalgebra of a chicken population (for short ECCP) is a $(n+1)$-dimensional $K$-coalgebra $C$ with (natural) basis $\mathcal{B}=\left\{h_{1}, \ldots, h_{n}, r\right\}$ and comultiplication given by:

$$
\begin{aligned}
& \Delta\left(h_{i}\right)=\sum_{j=1}^{n} \frac{1}{2} \beta_{j}^{i}\left(h_{j} \otimes r+r \otimes h_{j}\right), \quad i=1, \ldots, n ; \\
& \Delta(r)=\sum_{j=1}^{n} \frac{1}{2} \beta_{j}\left(h_{j} \otimes r+r \otimes h_{j}\right) .
\end{aligned}
$$

Proposition 4.2. ECCPs are cocommutative.

Proof. If follows straightforwardly from Definition 4.1.

Following [8] the comultiplication constants of any ECCP $C$ can be arranged into a cubic $(n+1) \times(n+1) \times(n+1)$ matrix $\widetilde{P}$, that unfolded by its frontal slices (see [12]) can be written as:

$$
\widetilde{P}=\left(\widetilde{P}_{:: 1}|\cdots| \widetilde{P}_{:: n} \mid \widetilde{P}_{:: n+1}\right)
$$


where $($ for $i=1, \ldots, n)$

$$
\widetilde{P}_{:: i}=\frac{1}{2}\left(\begin{array}{ccc|c}
0 & \cdots & 0 & \beta_{1}^{i} \\
\vdots & \ddots & \vdots & \vdots \\
0 & \cdots & 0 & \beta_{n}^{i} \\
\hline \beta_{1}^{i} & \cdots & \beta_{n}^{i} & 0
\end{array}\right) \quad \text { and } \quad \widetilde{P}_{:: n+1}=\frac{1}{2}\left(\begin{array}{ccc|c}
0 & \cdots & 0 & \beta_{1} \\
\vdots & \ddots & \vdots & \vdots \\
0 & \cdots & 0 & \beta_{n} \\
\hline \beta_{1} & \cdots & \beta_{n} & 0
\end{array}\right) \text {. }
$$

However, here as in [13] in the case of algebras, this cubic matrix can be replaced by a simpler rectangular $n \times(n+1)$ matrix of the form (up to a scalar $\frac{1}{2}$ ):

$$
P=[A \mid b]=\left(\begin{array}{ccc|c}
\beta_{1}^{1} & \cdots & \beta_{1}^{n} & \beta_{1} \\
\vdots & \ddots & \vdots & \vdots \\
\beta_{n}^{1} & \cdots & \beta_{n}^{n} & \beta_{n}
\end{array}\right)
$$

Definition 4.3. Given an ECCP $C$ we refer to the $n \times(n+1)$ matrix $P=[A \mid b]$ to be its associated structure matrix.

Remark 1. The matrix $P=[A \mid b]$ is close to be (up to a scalar $\frac{1}{2}$ ) the accompanying matrix (see $[10]$ ) of the cubic matrix $\widetilde{P}$. Indeed, being ECCPs cocommutative (see Proposition 4.2$)$, the cubic matrix $\widetilde{P}$ is $(1,2)$-symmetrical, so that their accompanying matrices coincide (a proof similar to that of $[10$, Lemma 2] works here). Thus we have:

$$
\widetilde{P}_{(i)}=\widetilde{P}_{(j)}=\frac{1}{2}\left(\begin{array}{ccc|c}
\beta_{1}^{1} & \cdots & \beta_{1}^{n} & \beta_{1} \\
\vdots & \ddots & \vdots & \vdots \\
\beta_{n}^{1} & \cdots & \beta_{n}^{n} & \beta_{n} \\
\hline \sum_{l=1}^{n} \beta_{l}^{1} & \cdots & \sum_{l=1}^{n} \beta_{l}^{n} & \sum_{l=1}^{n} \beta_{l}
\end{array}\right)
$$

Then (up to scalar $\frac{1}{2}$ ) $P=[A \mid b]$ is a submatrix of the accompanying matrices of $\widetilde{P}$.

Proposition 4.4. ECCPs are not in general coassociative.

Proof. Consider the $(2+1)$-dimensional ECCP $C$ with natural basis $\left\{h_{1}, h_{2}, r\right\}$ and comultiplication given by

$$
\begin{aligned}
& \Delta\left(h_{1}\right)=\frac{1}{2}\left(h_{2} \otimes r+r \otimes h_{2}\right) \\
& \Delta\left(h_{2}\right)=\sum_{j=1}^{2} \frac{1}{2} \beta_{j}^{2}\left(h_{j} \otimes r+r \otimes h_{j}\right), \quad \text { for arbitrary } \beta_{1}^{2}, \beta_{2}^{2} \in K \\
& \Delta(r)=\frac{1}{2}\left(h_{1} \otimes r+r \otimes h_{1}\right) .
\end{aligned}
$$

Now it is a straightforward checking to prove that $(\Delta \otimes i d) \Delta(r) \neq(i d \otimes \Delta) \Delta(r)$. Hence $C$, with the given comultiplication, is not coassociative.

Proposition 4.5. ECCPs are not counital.

Proof. Let $C$ be an ECCP and assume $\varepsilon: C \rightarrow K$ is a counit for $C$. Write then $\varepsilon\left(h_{i}\right)=$ $\alpha_{i}, i=1, \ldots, n$ and $\varepsilon(r)=\rho$ for the elements of a natural basis $\mathcal{B}=\left\{h_{1}, \ldots, h_{n}, r\right\}$ 
of $C$. Then applying the counit condition $(i d \otimes \varepsilon) \Delta=(\varepsilon \otimes i d) \Delta=i d$ to the natural basis elements we get:

$$
\begin{array}{lr}
\beta_{i} \rho=0, & i=1, \ldots, n, \\
\beta_{1} \alpha_{1}+\cdots+\beta_{n} \alpha_{n}=2, & \\
\beta_{j}^{i} \rho=0, & i \neq j, i, j=1, \ldots, n, \\
\beta_{i}^{i} \rho=2, & i=1, \ldots, n, \\
\beta_{1}^{i} \alpha_{1}+\cdots+\beta_{n}^{i} \alpha_{n}=0, & i=1, \ldots, n .
\end{array}
$$

By (4) we have $\rho \neq 0$. But if $\rho \neq 0$, then by (1) $\beta_{i}=0$ for all $i=1, \ldots, n$ which contradicts (2). As a result there is no counit for $C$.

Theorem 4.6. Any nontrivial character $\phi$ of an ECCP C is of the form $\phi\left(h_{i}\right)=\alpha_{i}$, $i=1, \ldots, n$ and $\phi(r)=\rho$, with $\rho \neq 0$ and such that $a^{T}=\left(\alpha_{1}, \ldots, \alpha_{n}\right)$ satisfies

$$
\begin{aligned}
& a^{T} b=1 \\
& a^{T} A=\frac{1}{\rho} a^{T}
\end{aligned}
$$

where $P=[A \mid b]$ is the $n \times(n+1)$ matrix associated to $C$.

Proof. Let $\phi \in \operatorname{Hom}_{K}(C, K)=C^{*}$ be a character of $C$ and write $\phi\left(h_{i}\right)=\alpha_{i}$, $i=1, \ldots, n$ and $\phi(r)=\rho$. Then as a result of $(\phi \otimes \phi) \Delta=\phi$ we obtain:

$$
\begin{aligned}
& \left(\beta_{1} \alpha_{1}+\cdots+\beta_{n} \alpha_{n}\right) \rho=\rho \\
& \left(\beta_{1}^{i} \alpha_{1}+\cdots+\beta_{n}^{i} \alpha_{n}\right) \rho=\alpha_{i}, \quad i=1, \ldots, n .
\end{aligned}
$$

Thus, if $\phi$ is a nontrivial character, necessarily, $\rho \neq 0$ and then $a^{T}=\left(\alpha_{1}, \ldots, \alpha_{n}\right)$ satisfies conditions $a^{T} b=1$ and $a^{T} A=\frac{1}{\rho} a^{T}$.

The connection between ECCPs introduced here and EACPs given in [4] goes through the dual structures. (We refer the reader to [1, Theorem 2.3.14] for a more general result on the duality between algebras and coalgebras.)

Theorem 4.7. Let $C$ be an $E C C P$. Then $C^{*}$ with the inherited algebra structure is an $E A C P$.

Proof. Let $\mathcal{B}=\left\{h_{1}, \ldots, h_{n}, r\right\}$ be a natural basis of the ECCP $C$ and let $\mathcal{B}^{*}=$ $\left\{h_{1}^{*}, \ldots, h_{n}^{*}, r^{*}\right\}$ be the dual basis of $C^{*}$ (i.e. $h_{i}^{*}\left(h_{j}\right)=\delta_{i j}, r^{*}(r)=1$ and $h_{i}^{*}(r)=$ $r^{*}\left(h_{i}\right)=0$ for all $\left.i, j=1, \ldots, n\right)$. Then it follows from Example 3.1 that the multiplication in $C^{*}$ for the basis elements of $\mathcal{B}^{*}$ is $h_{i}^{*} h_{j}^{*}=0=r^{*} r^{*}, i \neq j$, and

$$
r^{*} h_{i}^{*}=h_{i}^{*} r^{*}=\frac{1}{2}\left(\sum_{j=1}^{n} \beta_{i}^{j} h_{j}^{*}+\beta_{i} r^{*}\right), \quad i=1, \ldots, n .
$$

Hence $C^{*}$ is an EACP with natural basis $\mathcal{B}^{*}$. 
Theorem 4.8. Let $A$ be an $E A C P$ with natural basis $\mathcal{B}=\left\{h_{1}, \ldots, h_{n}, r\right\}$. Then $C=$ $A^{*}$ with the inherited coalgebra structure is an ECCP w.r.t. the natural basis $\mathcal{B}^{*}=$ $\left\{h_{1}^{*}, \ldots, h_{n}^{*}, r^{*}\right\}$.

Proof. Write $h_{i} r=r h_{i}=\frac{1}{2} \sum_{j=1}^{n}\left(a_{i j} h_{j}+b_{i} r\right)$ for the multiplication in the EACP $A$, $i=1, \ldots, n$, and zero otherwise. Then $C=A^{*}$ inherits a coalgebra structure (see [15]) such that the comultiplication for the elements of $\mathcal{B}^{*}$ is:

$$
\begin{aligned}
& \Delta\left(h_{i}^{*}\right)=\frac{1}{2} \sum_{j=1}^{n} a_{i j}\left(h_{j}^{*} \otimes r^{*}+r^{*} \otimes h_{j}^{*}\right), \\
& \Delta\left(r^{*}\right)=\frac{1}{2} \sum_{j=1}^{n} b_{j}\left(h_{j}^{*} \otimes r^{*}+r^{*} \otimes h_{j}^{*}\right) .
\end{aligned}
$$

As considered in [12] for general genetic coalgebras, here we will settle the structure of ECCPs in terms of those subcoalgebras of the chicken population.

Definition 4.9. Let $C$ be an ECCP $C$ (with natural basis $\mathcal{B}$ ). Then:

(i) A subcoalgebra $D$ of $C$ is an evolution subcoalgebra of the chicken population $C$ if $D$ is of the form $D=\operatorname{span}_{K}\left(h_{i}, r \mid i \in \Lambda\right), \emptyset \neq \Lambda \subseteq\{1, \ldots, n\}$.

(ii) The ECCP $C$ is said to be a simple ECCP is it has no proper evolution subcoalgebras of the chicken population (assuming also comultiplication is not trivial).

Remark 2. Any evolution subcoalgebra $D$ of a chicken population $C$ is also an ECCP itself, with the same roaster and a smaller (but not empty) group of hens. Note that this comes out from the nonempty assumption on the index set $\Lambda$, and makes $D$ to retain the defining property of chicken populations. The existence of only one roaster in chicken populations makes all evolution coalgebras of chicken populations irreducible (as chicken populations) since having just one roaster, it becomes impossible to decompose any chicken population into the direct sum of two such subpopulations (each having its own roaster).

Left, right and two-sided coideals of chicken populations can be defined similarly. Note, however, that as coideals are not necessarily subcoalgebras, their bases (as spanning subsets) do not need to contain a chicken subpopulation. A first approach to the structure of ECCPs was given in [13], where it was settled that any $(n+1)$ - dimensional ECCP is the direct sum of an $n$-dimensional and a 1-dimensional coideals. However these coideals are not coideals of the chicken population. Indeed such decomposition was given in term of evolution coideals (i.e. coideals spanned by subsets of the natural basis) but not of the chicken population.

Proposition 4.10. Let $C$ be an ECCP with natural basis $\mathcal{B}=\left\{h_{1}, \ldots, h_{n}, r\right\}$. Then $D=\operatorname{span}_{K}\left(h_{i}, r \mid i \in \Lambda\right), \Lambda \subseteq\{1, \ldots, n\}$ is an evolution subcoalgebra of the chicken population $C$ if and only if $D^{*}$ is an evolution subalgebra of the EACP $C^{*}$. Hence $C$ is simple (as ECCP) if and only if $C^{*}$ is irreducible (as EACP).

Proof. It follows from Theorem 4.7 and Theorem 4.8. For the last assertion see [7, Definition 4.5] 


\section{The structure of evolution coalgebras of chicken populations.}

In this section we introduce the notion of digraph attached to an ECCP and use it to characterize simple ECCPs. In what follows we will assume that $C$ is an ECCP with natural basis $\mathcal{B}=\left\{h_{1}, \ldots, h_{n}, r\right\}$ and comultiplication as given in Definition 4.1. We refer the reader to [11] for basic notions on digraphs attached to coalgebras with genetic realization (see also [3] for digraphs attached to evolution algebras).

Definition 5.1. We define the digraph attached to an ECCP $C$ relative to $\mathcal{B}$ to be the (directed) graph $\Gamma(C, \mathcal{B})=(V, E)$ with set of vertices $V=\{0,1, \ldots, n\}$ and set of directed edges (or arcs):

$$
E=\left\{(k, i) \in V \times V \mid \beta_{i}^{k} \neq 0, i, k \neq 0\right\} \bigcup\left\{(0, i) \in V \times V \mid \beta_{i} \neq 0, i \neq 0\right\} .
$$

Remark 3. (i) Vertices $1, \ldots, n$ represent the "hens", while vertex 0 represents the roaster $r$. Clearly this vertex is a source, with arcs only existing but never entering at it.

(ii) $\Gamma(C, \mathcal{B})$ may contain loops (i.e. arcs of the form $u u$ for some $u \in V$ ) only for those vertices in $V-\{0\}$.

(iii) $\Gamma(C, \mathcal{B})$ is naturally weighted by the matrix $P=[A \mid b]$ associated to $C$. Indeed it suffices to consider $\omega(k, i)=\beta_{i}^{k}$ and $\omega(0, i)=\beta_{i}$ for all $i, k=1, \ldots, n$. Then the map $\omega: E \rightarrow K$ defines a weighting on $\Gamma(C, \mathcal{B})$.

Example 5.2. Consider a $(2+1)$-dimensional ECCP $C$ with natural basis $\mathcal{B}=$ $\left\{h_{1}, h_{2}, r\right\}$. Then $\Gamma(C, \mathcal{B})$ is

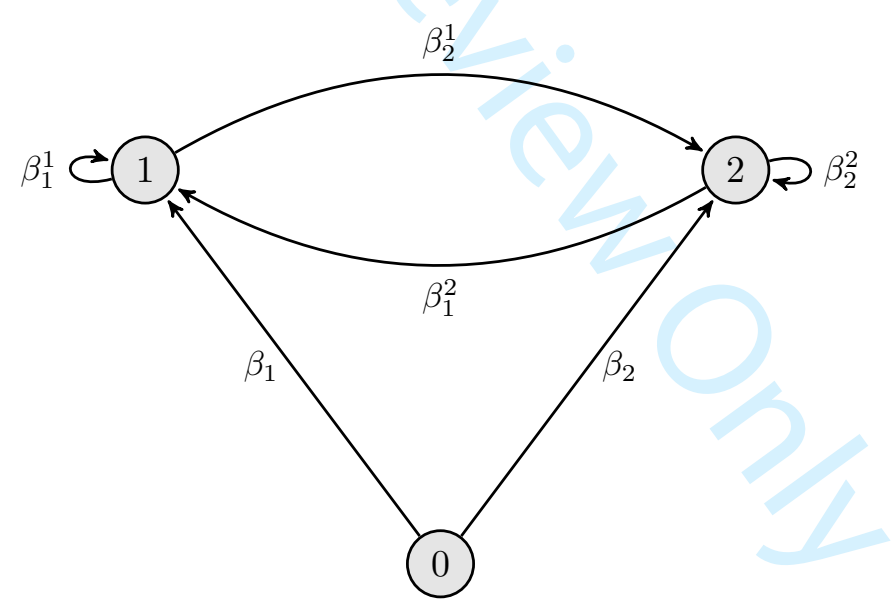

To be more accurate the digraph associated to any $(2+1)$-dimensional ECCP is a subdigraph of the digraph $\Gamma(C, \mathcal{B})$ above, after taking into account that the vanishing of any of the comultiplication structure constants should lead us to discard the corresponding arc.

Definition 5.3. We define the $h$-subdigraph $\Gamma_{h}(C, \mathcal{B})$ of $\Gamma(C, \mathcal{B})$ to be the subdigraph of $\Gamma(C, \mathcal{B})$ with vertices $V_{h}=V-\{0\}$ and $\operatorname{arcs}$

$$
E_{h}=\left\{(k, i) \in V \times V \mid \beta_{i}^{k} \neq 0, i, k \neq 0\right\} .
$$


Proposition 5.4. $\Gamma_{h}(C, \mathcal{B})$ is an induced subdigraph of $\Gamma(C, \mathcal{B})$. Moreover $\omega$ induces a weighting in $\Gamma_{h}(C, \mathcal{B})$.

Proof. Recall that a subgraph $H$ of a graph $G$ is an induced subgraph if all edges in $G$ linking vertices in $H$ belong to $H$. The fact that the restriction of $\omega$ to $\Gamma_{h}(C, \mathcal{B})$ induces a weighting on $\Gamma_{h}(C, \mathcal{B})$ is straightforward.

Example 5.5. Let $\Gamma(C, \mathcal{B})$ be as in Example 5.2. Then the corresponding $h$ subdigraph $\Gamma_{h}(C, \mathcal{B})$ is:

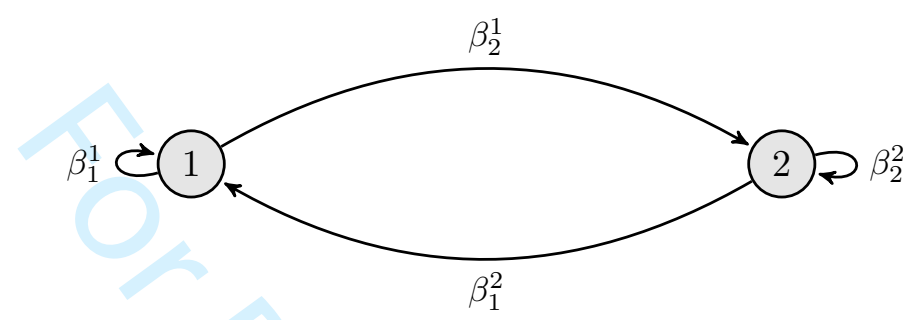

Let us denote $\Gamma=\Gamma(C, \mathcal{B})$ and by $\Gamma_{h}$ the corresponding $h$-subdigraph of $\Gamma$. We recall that for any $i \in V, N_{\Gamma}(i)=\{j \in V \mid(i, j) \in E\}$ denotes the set of all neighbors of $i$ in $\Gamma$. Note that for all $i \in V$, we have $N_{\Gamma}(i) \subseteq V-\{0\}$. Moreover $N_{\Gamma}(i)=N_{\Gamma_{h}}(i)$, for all $i \in V-\{0\}$, as a result of being $\Gamma_{h}$ an induced subgraph of $\Gamma$ (see Proposition 5.4).

Following [11] it is not difficult to establish a correspondence between evolution subcoalgebras of the chicken population $C$ and certain induced subdigraphs of $\Gamma(C, \mathcal{B})$.

Proposition 5.6. Let $C$ be an ECCP with natural basis $\mathcal{B}$ and let $D=\operatorname{span}_{K}\left(h_{i}, r \mid\right.$ $i \in \Lambda), \Lambda \subseteq\{1, \ldots, n\}$, be an evolution subcoalgebra of the chicken population. Then $\Gamma_{D}=\left(V_{D}, E_{D}, \omega_{D}\right)$ where:

(i) $V_{D}=\{0, i \mid i \in \Lambda\}$,

(ii) $E_{D}=\left\{(k, i) \mid \beta_{i}^{k} \neq 0, i, k \in \Lambda\right\} \bigcup\left\{(0, i) \mid \beta_{i} \neq 0, i \in \Lambda\right\}$, (iii) $\omega_{D}: E_{D} \rightarrow K$ given by $\omega_{D}(k, i)=\beta_{i}^{k}$ and $\omega_{D}(0, i)=\beta_{i}$, for all $i, k \in \Lambda$,

is a weighted induced subdigraph of $\Gamma(C, \mathcal{B})$ with $\omega_{D}=\omega_{\mid D}$. Conversely, for any induced subdigraph $\Gamma^{\prime}$ of of $\Gamma(C, \mathcal{B})$ with $0 \in V\left(\Gamma^{\prime}\right)$ and such that $N_{\Gamma^{\prime}}(k)=N_{\Gamma(C, \mathcal{B})}(k)$ for all $k \in V\left(\Gamma^{\prime}\right)$, the linear span $D=\operatorname{span}_{K}\left(k \in V\left(\Gamma^{\prime}\right)\right)$ is an evolution subcoalgebra of the chicken population $\mathcal{C}$.

Proof. A proof similar to that of [11, Proposition 6.1, Corollary 6.2] works here to prove that $\Gamma_{D}$ is a weighted induced subdigraph of $\Gamma(C, \mathcal{B})$. The second assertion follows as in [11, Theorem 6.3].

Example 5.7. Consider the following (1+1)-dimensional ECCPs:

(i) $C_{1}$ with natural basis $\mathcal{B}=\{h, r\}$ and comultiplication:

$$
\begin{aligned}
& \Delta(h)=h \otimes r+r \otimes h, \\
& \Delta(r)=0 ;
\end{aligned}
$$


(ii) $C_{2}$ with natural basis $\mathcal{B}=\{h, r\}$ and comultiplication:

$$
\begin{aligned}
& \Delta(h)=\frac{1}{2}(h \otimes r+r \otimes h), \\
& \Delta(r)=\frac{1}{2}(h \otimes r+r \otimes h) .
\end{aligned}
$$

Then the corresponding attached digraphs $\Gamma_{i}, i=1,2$, are, respectively:
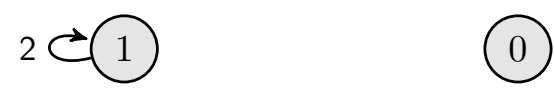

and

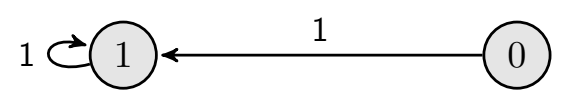

We note here that duals of ECCPs $C_{1}$ and $C_{2}$ above are the EACPs appearing in [4, Proposition 6.1] (see also [14, Propositon 4.1]).

A (directed) walk $u-v$ (of length $k$ ) in a digraph is a sequence of vertices $u=$ $u_{0} u_{1} \cdots u_{k}=v$, with $u_{i-1}$ adjacent to $u_{i}$ (i.e. $u_{i-1} u_{i}$ is an arc). A digraph is strongly connected if there are $u-v$ and $v-u$ walks for all vertices $u, v$. Otherwise the digraph is said to be disconnected. If only the underlying non-directed graph is connected the digraph is weakly connected.

Proposition 5.8. Digraphs attached to a chicken population are not strongly connected.

Proof. It suffices to note that, being vertex 0 a source, $\Gamma(C, \mathcal{B})$ contains no walks finishing at vertex 0 .

In what follows we will denote by $\Gamma$ the digraph $\Gamma(C, \mathcal{B})$ attached to the ECCP $C$ w.r.t the natural basis $\mathcal{B}=\left\{h_{1}, \ldots, h_{n}, r\right\}$ and by $\Gamma_{h}$ its induced $h$-subdigraph (see Definition 5.3).

Theorem 5.9. Let $C$ be an $E C C P$ with natural basis $\mathcal{B}$. Assume that $\Delta(x) \neq 0$ for all $x \in \mathcal{B}$. Then, $C$ is simple as ECCP if and only if $\Gamma_{h}$ is strongly connected.

This theorem is based on the following technical lemmas whose proofs work similarly to those of the corresponding results given in [11] for arbitrary genetic coalgebras.

Lemma 5.10. The following statements hold for all $i, k \in\{1, \ldots, n\}$ :

(i) $i \in N_{\Gamma}(k) \Rightarrow h_{i} \in \operatorname{subcoalg}\left(h_{k}\right)$.

(ii) $i \in N_{\Gamma}(0) \Rightarrow h_{i} \in \operatorname{subcoalg}(r)$.

Here subcoalg $(x)$ denotes the smallest (ordinary) subcoalgebra of $C$ containing the element $x$ (see 3.2).

Proof. Similar to [11, Proposition 6.5] and [11, Corollary 6.8].

Lemma 5.11. Let $i-j, j \neq 0$, be a directed walk in $\Gamma$ of length $s \geq 2$ for some $i, j \in\{0,1, \ldots, n\}$. Then: 
(i) If $i=0$, then $j \neq i$ and there exist indexes $j_{1}, j_{2}, \ldots, j_{s-1} \in\{1, \ldots, n\}$ such that $\beta_{j_{1}} \beta_{j_{2}}^{j_{1}} \ldots \beta_{j_{s-1}}^{j_{s-2}} \beta_{j}^{j_{s-1}} \neq 0$.

(ii) If $i \neq 0$, then there exist indexes $j_{1}, j_{2}, \ldots, j_{s-1} \in\{1, \ldots, n\}$ such that $\beta_{j_{1}}^{i} \beta_{j_{2}}^{j_{1}} \ldots \beta_{j_{s-1}}^{j_{s-2}} \beta_{j}^{j_{s-1}} \neq 0$.

Conversely if such a series of indexes exists in $\{1, \ldots, n\}$, then there exists a $i-j$ walk in $\Gamma$.

Proof. The proof works as that of [11, Lemma 6.4].

Lemma 5.12. Let $i, j \in\{0,1, \ldots, n\}$. If there exists $a i-j$ walk in $\Gamma$ then $j \neq 0$ and $h_{j} \in \operatorname{subcoalg}\left(h_{i}\right)$. In particular, $h_{j} \in \operatorname{subcoalg}\left(h_{i}\right)$ for all $0 \neq j \in N_{\Gamma}(i)$. (Write here $h_{0}=r$.)

Proof. See the proof of [11, Corollary 6.6] and [11, Corollary 6.8].

Proposition 5.13. Any subcoalgebra of $C$ generated by a natural basis element (i.e. any subcoalgebra of $C$ of the form subcoalg $(x), x \in \mathcal{B})$ with $\Delta(x) \neq 0$ contains an evolution subcoalgebra of the chicken population $C$.

Proof. Similar to the proof of [11, Proposition 6.9]. We remark that here assumption $\Delta(x) \neq 0$ plays the role of the genetic realization assumption appearing in [11].

Remark 4. Note that any natural basis element $x \in \mathcal{B}$ with trivial comultiplication, i.e. such that $\Delta(x)=0$, is a source in $\Gamma$.

Finally we have all technical requirements to cope with Theorem 5.9, whose proof follows the main guidelines of [11, Theorem 6.10] and [11, Theorem 6.11].

Proof. Proof of Theorem 5.9. Assume first that $C$ is simple as ECCP. We claim that there exists a $i-j$ walk in $\Gamma_{h}$ for all $i, j \in\{1, \ldots, n\}$.

Let subcoalg $\left(h_{i}\right)$ be the (ordinary) subcoalgebra of $C$ generated by $h_{i}$. Then, since $\Delta\left(h_{i}\right) \neq 0$, subcoalg $\left(h_{i}\right)$ contains an evolution subcoalgebra of the chicken population $C$. But being $C$ simple, as chicken population, we have $C \subseteq \operatorname{subcoalg}\left(h_{i}\right)$. Then

$$
S=\left\{p \mid p \in N_{\Gamma}(k) \text { for some } h_{k} \in \operatorname{subcoalg}\left(h_{i}\right)\right\}=\{1, \ldots, n\} .
$$

Thus there exists a series of indexes $k_{0}=i, k_{1}, \ldots, k_{l}=j(l \geq 1)$ with $k_{m} \in N_{\Gamma}\left(k_{m-1}\right)$, $m=1, \ldots, l$, so that $k_{m-1}$ is adjacent to $k_{m}$ giving rise to a $i-j$ walk in $\Gamma_{h}$. Hence $\Gamma_{h}$ is strongly connected.

Conversely, suppose now that $\Gamma_{h}$ is strongly connected and let $D$ be a proper evolution subcoalgebra of the chicken population $C$. Write, reordering the basis if necessary, $D=\operatorname{span}_{K}\left(h_{1}, \ldots, h_{t}, r\right)$ for some $1 \leq t<n$. Take $k_{0}$, such that $t<k_{0} \leq n$. Being $\Gamma_{h}$ strongly connected, there exists a $t-k_{0}$ walk in $\Gamma_{h}$ implying that $k_{0} \in \operatorname{subcoalg}\left(h_{t}\right) \subseteq D$, contradicting that $t<k_{0}$. Hence $C$ is simple as ECCP.

We conclude by remarking that Theorem 5.9 is not only a simplicity criteria for ECCPs. Indeed as a result of Theorem 4.7, Theorem 4.8 and Proposition 4.10 it also provides us a new tool for studying the structure of EACPs. 


\section{Acknowledgement(s)}

Partially supported by the Spanish Ministerio de Economía y Competitividad and FEDER, grant MTM2017-83506-C2-1-P (AEI/FEDER, UE).

\section{References}

[1] E. Abe, Hopf algebras, Cambridge University Press, Cambridge, New York, 1980.

[2] A. Dzhumadil'daev, B.A. Omirov, U. A. Rozikov, On a class of evolution algebras of "chicken" population, International Journal of Mathematics 25 (8) (2014) 19 pp.

[3] A. Elduque, A., A. Labra, Evolution algebras and graphs, J. Algebra Appl. 14 (7) (2015) $10 \mathrm{pp}$.

[4] A. Labra, M. Ladra, U. A. Rozikov, An evolution algebra in population genetics, Linear Algebra Applications 457 (2014) 348-362.

[5] M. Ladra, U. A. Rozikov, Evolution algebra of a bisexual population, J. Algebra 378 (2013) 153-172.

[6] M. Ladra, U. A. Rozikov, Evolution algebra of a "chicken" population, arXiv:1307:4916 (2015).

[7] B. A. Omirov, U. A. Rozikov, On subalgebras of an evolution algebra of a "chicken" population, J. Algebra Relat. Topics 5(2) (2017) 13-24.

[8] I. Paniello, Stochastic matrices arising from genetic inheritance, Linear Algebra and its Applications 434 (2011) 791-800.

[9] I. Paniello, Marginal distributions on genetic coalgebras, J. Math. Biology 68 (5) (2014) 1071-1087.

[10] I. Paniello, On evolution operators of genetic coalgebras, J. Math. Biology 74 (1-2) (2017) 149-168.

[11] I. Paniello, Backwards genetic inheritance through coalgebra-graphs, Linear and Multilinear Algebra 65 (5) (2017) 943-961.

[12] I. Paniello, Genetic coalgebras and their cubic stochastic matrices, J. Algebra Appl. 16 (11) (2017) $22 \mathrm{pp}$.

[13] I. Paniello, Evolution coalgebras, to appear in Linear and Multilinear Algebra DOI $10.1080 / 03081087.2018 .1460795$.

[14] U. A. Rozikov, Sh. Murodov, Chain of evolution algebras of chicken population, Linear Algebra Applications 450 (2014) 180-201.

[15] M. E. Sweedler, Hopf Algebras, Mathematics Lecture Note Series, W. A. Benjamn, Inc. New York, 1969.

[16] J. P. Tian, Evolution algebras and their applications, Lecture Notes in Biomathematics 36, Springer, Berlin, 2008.

[17] J. Tian, B-L. Li, Coalgebraic structure of genetic inheritance, Mathematical Biosciences and Engineering 1 (2) (2004) 243-266. 